\title{
Protéines de choc thermique, transport des protéines dans le noyau et oncogenèse
}

Des protéines de choc thermique (HSP) interviennent dans le transfert des protéines du cytoplasme où elles sont synthétisées, vers la membrane plasmique ou vers les organites : mitochondries, réticulum endoplasmique, lysosomes ( $\mathrm{m} / \mathrm{s}$ vol. 7, p. 496) [1]. Les protéines de choc thermique de 70 kDa (HSP70) s'associent aux chaînes polypeptidiques en cours de biosynthèse. Pour franchir les parois des organites, les protéines doivent être maintenues dépliées, l'interaction avec des protéines de choc thermique HSP70 stabilise ce dépliement. Cette interaction est ensuite rompue avec hydrolyse d'ATP. Des expériences plus récentes ont montré que des protéines de choc thermique de la même famille interviennent aussi dans le transfert des protéines du cytoplasme vers le noyau $[2,3]$. Rappelons d'abord que les protéines de choc thermique ont été appelées comme telles pour des raisons phénoménologiques et historiques, car elles sont codées par des familles de gènes dont l'expression est stimulée par les traitements qui accélèrent la dénaturation des protéines (dont le choc thermique) [4-6]. La dénaturation est une conséquence inappropriée d'un dépliement des chaînes polypeptidiques. Dans la cellule, la dénaturation n'est pas forcément irréversible $[7,8]$, et il est probable que des protéines de choc thermique catalysent la renaturation des protéines. En effet, in vitro, les protéines de choc thermique ont tendance à s'associer aux protéines dénaturées ; cette association réversible facilite la renaturation suivant des processus dont l'efficacité est fortement améliorée lorsqu'on fait agir un " cocktail " de protéines de choc thermique et d'ATP [9]. Précisons aussi que des protéines toutes les cellules en croissance "normale ". L'interaction des protéines de choc thermique de $70 \mathrm{kDa}$ (HSP70) avec le noyau est connue depuis une dizaine d'années. A la suite d'un choc thermique, les HSP70 s'accumulent dans le noyau, en particulier au niveau des nucléoles [10, 11]. Elles retournent dans le cytoplasme pendant la récupération des cellules à une température normale de croissance. La morphologie des nucléoles est affectée par le choc thermique et les protéines HSP70 contribuent à la restauration d'une morphologie " normale " [12].

Une accumulation nucléaire d'une HSP70 dans les conditions de croissance "normale" se produit aussi à certaines phases du cycle cellulaire : pendant la phase $\mathrm{S}$ de réplication de l'ADN et la mitose [13]. D'ailleurs, on a observé au cours du cycle, des variations dans la présence de protéines capables de former des associations stables avec cette HSP70 [14]. Dans l'ovocyte de xénope, les HSP70 entrent et sortent du noyau en permanence [15]. Ce comportement évoque une fonction de transport. Et en effet, l'implication des HSP70 dans le transport des protéines vers le noyau a été montré dans une série d'expériences récentes utilisant la reconstitution du transport nucléaire avec des extraits de cellule HeLa [3]. Le transport nucléaire ne s'effectue pas avec des extraits dont on a retiré les HSP70 par immunoadsorption, mais la capacité de transport est restaurée en rajoutant des HSP70 purifiées aux extraits carencés. Il existe aussi des arguments génétiques qui suggèrent que des protéines apparentées aux protéines de choc thermique contribuent au transport nucléaire des protéines. L'antigène $\mathrm{T}$ du virus SV40 possède un peptide signal d'une dizaine d'acides aminés qui commande la localisation nucléaire [16]. Chez la levure, on a donc recherché des mutations supprimant le transport d'une protéine chimérique possédant le signal d'adressage nucléaire de l'antigène $T$. Le gène NPL1, allèle du gène SEC63, a été isolé [17]. Ce gène code pour une protéine présentant une homologie de séquence significative avec la protéine de choc thermique bactérienne, DnaJ. Le gène $S E C 63$ avait été caractérisé auparavant ; il est nécessaire à la sécrétion des protéines à travers le réticulum endoplasmique [18]. Utilisant une approche différente, Caplan et al. ont recherché des gènes qui codent pour les constituants des pores de la matrice nucléaire. Ainsi, ils ont isolé le gène, YDJ1 (distinct de NPL1), qui code pour une protéine présentant elle aussi une bonne homologie de séquence avec DnaJ et dont le produit se retrouve autour du noyau et associé au réticulum endoplasmique [19]. Les protéines de choc thermique ont été extrêmement conservées à travers l'évolution, et la plupart des propriétés fonctionnelles des protéines bactériennes se retrouvent chez leurs homologues eucaryotes [5]. La HSP70 bactérienne, DnaK, n'agit pas seule, elle a des auxiliaires: DnaJ et GrpE. DnaJ coopère directement avec DnaK pour fixer les polypeptides dépliés, puis GrpE catalyse le transfert de ceux-ci sur d'autres cibles, par exemple GroEL (HSP60) [9]. La réalité physiologique de cette coopération est illutrée par le fait que, chez $E$. Coli, DnaK et DnaJ sont codées par le même opéron, et que, chez d'autres bactéries, DnaK, DnaJ et GrpE font partie d'un seul et même opéron. Il est donc remarquable de constater que, chez les eucaryotes, les voies de translocation des pro- 


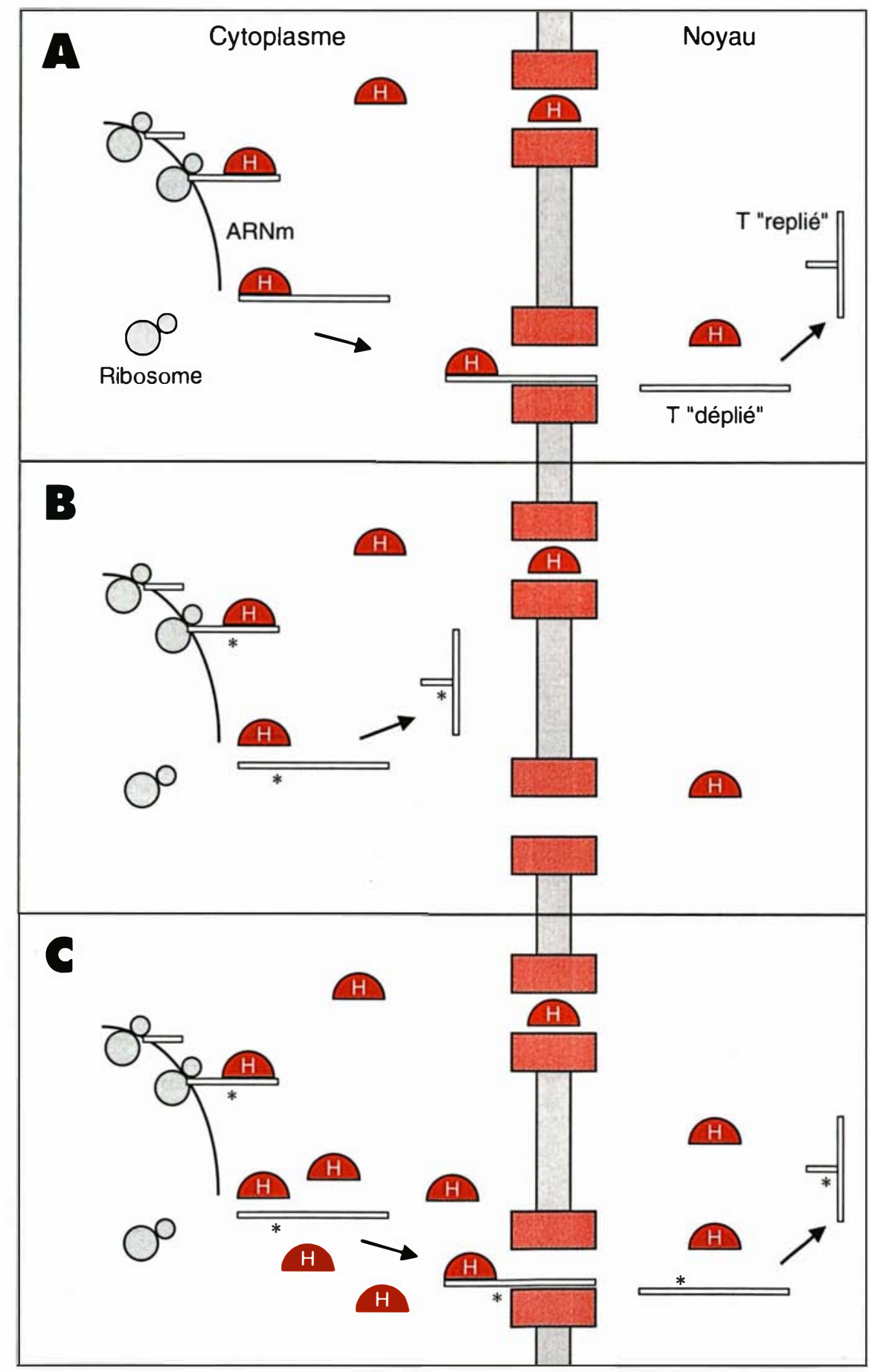

Figure 1. Interaction entre les protéines de choc thermique HSP70 et l'antigène $T$ du virus SV40. En A, nous avons schématisé l'interaction entre les HSP7O $(H)$ et un antigène $T$ fonctionnel (de type sauvage) ; l'interaction d'HSP7O avec l'antigène $T$ en cours de biosynthèse stabilise une conformation dépliée de I'antigène $T$, qui permet la translocation du cytoplasme où il est synthétisé, vers le noyau de la cellule. En B, l'interaction avec un antigène $T$ muté est instable, l'antigène $T$ adopte dans le cytoplasme une conformation incompatible avec la translocation. II n'est pas transformant. En C, la surproduction d'HSP7O stabilise la conformation dépliée de l'antigène $T$ muté, en déplaçant l'équilibre $T$ lié/T libre vers la forme liée. II est transformant.

$\mathrm{m} / \mathrm{s} n^{\circ} 7$, vol. 8, seplembre 92 téines vers le réticulum endoplasmique et vers le noyau utilisent les mêmes protéines homologues de DnaK et au moins deux protéines apparentées à DnaJ.

Un certain nombre de cellules tumorales synthétisent spontanément de fortes quantités de protéines de choc thermique [6, 20-26). Est-ce fortuit, ou bien cette surexpression peut-t-elle jouer un rôle dans la tumorigenèse? La surexpression concerne généralement les protéines de choc thermique de $90 \mathrm{kDa}$ qui sont impliquées dans le transport d'oncogènes membranaires (tyrosine kinases), des protéines du réticulum endoplasmique impliquées dans la sécrétion, et/ou les HSP70. Mais gardons-nous de trop généraliser : d'une part, la synthèse des protéines de choc thermique n'est pas stimulée dans tous les cas de transformation cellulaire ; d'autre part, la transformation inhibe la synthèse de certaines protéines de choc thermique ; c'est le cas de la HSP47 qui participe au transport de protéines secrétées comme le collagène [27].

Les HSP70 s'accumulent dans le noyau au cours de la phase de synthèse d'ADN (voir plus haut) et pendant la réplication de virus à $\mathrm{ADN}$ [28]. Une participation directe des protéines de choc thermique au processus réplicatif peut être envisagée puisque, chez E. Coli, l'homologue des HSP70 eucaryotes, DnaK, contribue à la réplication de l'ADN des phages et des plasmides [5].

Les protéines de choc thermique sontelles impliquées dans le transport nucléaire de protéines liées à la prolifération? Beaucoup d'oncogènes et d'antioncogènes viraux et cellulaires sont localisés dans le noyau, et une interaction avec les HSP70 a été trouvée pour plusieurs d'entre eux : les antigènes $\mathrm{mT}$ du virus polyome [29] et $\mathrm{T}$ du virus SV40 [30,31], les protéines E1A de l'adénovirus [32], Rel [33], p53 [34, 35] et Myc [36]. Des complexes stables ont pu être isolés dans un certain nombre de cas. En particulier, celui de la p53 dont l'extrémité N-terminale peut se lier aux HSP70 [37]. L'association p53 de mammifères/HSP70 de mammifère n'a pu être isolée qu'avec des p53 mutées et semble liée au phénotype oncogène des p53 mutées ( $m / s n^{\circ} 4$, vol. 6, 
p. 352). Par ailleurs, l'association p53 de xénope/HSP70 de mammifère ne s'observe qu'à des températures supérieures à $33^{\circ} \mathrm{C}$, ce qui suggérerait que la p53 ne forme des complexes

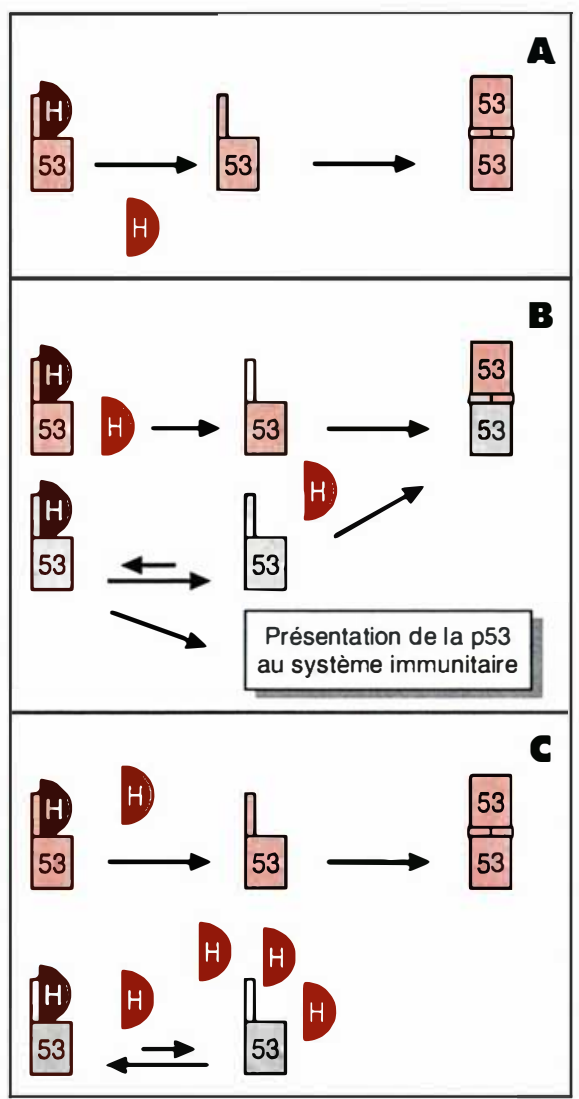

Figure 2. Interaction entre les protéines de choc thermique HSP7O et I'antigène tumoral p53. En A, la protéine p53 sauvage qui vient d'être synthétisée forme un complexe instable avec les HSP7O (H). En B, une protéine p53 mutée (grisé) est synthétisée en même temps que de la p53 sauvage. Le complexe HSP70/p53 mutée est relativement stable, ce qui augmente la durée de vie de la p53 et favorise la présentation de la p53 mutée au système immunitaire. Du fait de sa longue durée de vie, cette p53 mutée s'accumule et s'associe à la p53 sauvage en l'inactivant, d'où le phénotype oncogène dominant des p53 mutées. En C, la surproduction d'HSP7O défavoriserait la dissociation des complexes HSP70/p53 mutée et donc la formation de complexes p53 mutée/p53 sauvage d'où restauration du phénotype non transformant de la p53 sauvage endogène. stables avec les HSP70 que lorsqu'elle est légèrement dénaturée. La stabilité de cette interaction pourrait avoir deux conséquences importantes: (a) augmenter la durée de vie de la protéine p53 mutée; (b) contribuer à l'antigénicité de cette protéine qui a été d'abord caractérisée comme un antigène tumoral [38]. Cette dernière observation pourrait illustrer le rôle d'HSP70 dans la présentation des antigènes par le système majeur d'histocompatibilité de classe II [39].

L'intervention d'HSP70 dans le transport nucléaire d'un oncogène a été montrée dans un travail récent [2]. En effet, une mutation ponctuelle (Lys ${ }^{128}$ changée en $A s n^{128}$ ) dans la séquence d'adressage nucléaire de l'antigène T du SV40 abolit la localisation nucléaire du $\mathrm{T}$ et son pouvoir transformant. Cependant, la surexpression d'une HSP70 humaine dans des cellules murines restaure le phénotype nucléaire et transformant du $\mathrm{T}$. Il semblerait, en outre, qu'en surexprimant HSP70, on supprimerait le phénotype oncogène des p53 mutécs (travail de M. Oren cité dans la référence [6]). On peut supposer qu'en favorisant la formation de complexes HSP70/p53 mutée, les complexes p53 mutée/p53 sauvage seraient défavorisés, ce qui restaurerait la fonction normale de la p53 sauvage.

Ainsi, la surexpression des protéines de choc thermique pourrait contribuer, dans certains cas, à la transformation en facilitant le transport nucléaire d'oncogènes. Mais, dans d'autres cas, la formation de complexes avec les protéines de choc thermique pourrait au contraire, défavoriser la transformation

Olivier Bensaude

Groupe de biologie du stress cellulaire, génétique moléculaire, URA Cnrs 1302, Ecole normale supérieure, 46, nue d'Ulm, 75230 Paris Cedex 05, France.

\section{TIRÉS A PART}

O. Bensaude.

\section{RÉFÉRENCES}

1. Gething MJ, Sambrook J. Protein folding in the cell. Nature $1992 ; 355: 33-45$.

2. Jeoung DI, Chen S, Windsor J, Pollack RE. Human major HSP70 protein complements the localization and functional defects of cytoplasmic mutant SV40 T antigen in Swiss 3T3 mouse fibroblast cells. Genes Dev 1991 ; 5 : 2235-44.

3. Shi Y, Thomas JO. The transport of proteins into the nucleus requires the 70-kilodalton heat shock protein or its cytosolic cognate. $\mathrm{Mo}$ Cell Biol 1992 ; 12 : 2186-92.

4. Hightower LE. Heat shock, stress proteins, chapcrones, and protcotoxicity. Cell 1991; 66 : 191-7.

5. Ang D, Liberek DK, Skowwyra D, Zylicz $\mathrm{M}$, Georgopoulos C. Biological role and regulation of the universally conserved heat shock proteins. J Biol Chern 1991; 266 : 24233-36.

6. Morimoto RI. Heat shock: the role of transient inducible responses in cell damage, transformation, and differentiation. Cancer Cells $1991 ; 3$ : 295-301.

7. Dubois MF, Hovanessian AG, Bensaude O. Heat-shock-induced denaturation of proteins. Characterization of the insolubilization of the interferon-induced p68 kinase. $J$ Biol Chern 1991; 266 : 9707-11.

8. Pinto $\mathrm{M}$, Morange $\mathrm{M}$, Bensaude $\mathrm{O}$. Denaturation of proteins during heat shock. In vivo recovery of solubility and activity of reporter enzymes. I Biol Chern 1991; 266 : 13941-6.

9. Langer T, Lu C, Echols H, Flanagan J, Hayer MK, Hartl FU. Successive action of DnaK, DnaJ and GroEL along the pathway of chaperone-mediated protein folding. Nature 1992 ; 356 : 683-9.

10. Velazquez JM, Lindquist S. Hsp70 : nuclear concentration during environmental stress and cytoplasmic storage during recovery. Cell $1984 ; 36$ : 655-62.

11. Welch WJ, Feramisco JR. Nuclear and nucleolar localization of the 72,000 dalton heat shock protein in heat-shocked mammalian cells. J Biol Chem 1984; 259 : 4501-13.

12. Pelham HRB. Hsp70 accelerates the recovery of nucleolar morphology after heat shock $E M B O J 1984 ; 3$ : 3095-100.

13. Milarski KL, Morimoto RI. Expression of human HSP70 during the synthetic phase of the ccll cycle. Proc Natl Acad Sci USA 1986 ; 83 : 9517-21.

14. Milarski K Welch W Morimoto RI Cell cycle-dependent association of HSP70 with specific cellular proteins. J Cell Biol 1989 ; 108 : 413-23. 


\section{RÉFÉRENCES}

15. Mandell RB, Feldherr CM. Identification of two Hsp70-related xenopus oocytc proteins that are capable of recycling across the nuclear envclopc. J Cell Biol 1990 ; 111 : 1775-83.

16. Kalderon D, Roberts BL, Richardson WD, Smith AE. A short amino acid scquence able to spccify nuclear location. Cell 1984; 39 : 479-509.

17. Sadler I, Chiang A, Kurihara T, Rothblatt J, Way J, Silver P. A yeast gene important for protein assembly into the endoplasmic reticulum and the nuclcus has homology to DnaJ, an Escherichia coli heat shock protcin. J Cell Biol 1989 ; 109 : 2665-75.

18. Sanders SL, Whitficld KM, Vogel JP, Rose MD, Schekman RW. Scc61p and BiP dircctly facilitate polypeptide translocation into the ER. Cell $1992 ; 69$ : 353-65.

19. Caplan AJ, Douglas MG. Characterization of YDJ1 : a ycast homologuc of the bacterial DnaJ protcin. J Cell Biol 1991; 114 : 609-21.

20. Pouysségur J, Shiu RPC, Pastan I. Induction of two transformation-sensitive membrane polypcptides in normal fibroblasts by a block in glycoprotein synthesis or glucose deprivation. Cell 1977 ; 11: 941-7.

21. Bensaude $\mathrm{O}$, Morange M. Spontaneous high expression of heat-shock proteins in mousc cmbryonal carcinoma cells and ectoderm from day 8 mousc embryo. EMBO J $1983 ; 2: 173-7$.

22. Simon MC, Kitchener K, Kao HH, et al. Selective induction of human heat shock gene transcription by the adenovirus E1A gene products, including the $12 \mathrm{~S}$ E1A Product. Mol Cell Biol 1987 ; 7 : 2884-90.

23. La Thanguc NB, Latchman DS. A cellular protcin related to heat-shock protein 90 accumulates during herpes simplex virus infection and is overexpressed in transformed cells. Exp Cell Res 1988 ; 178 : 169-79.

24. Stoeckle MY, Sugano S, Hampc A, Vashishta A, Pellman D, Hanafusa $H$. 78-kilodalton glucose-regulated protein is induced in Rous sarcoma virus-transformed cells independently of glucose deprivation. Mol Cell Biol 1988 ; 8 : 2675-80.

25. Fuqua SA, Blum-Salingaros $\mathrm{M}, \mathrm{McGuire}$ WL. Induction of the estrogen-regulated " $24 \mathrm{~K}$ " protein by heat shock. Cancer Res $1989 ; 49: 4126-9$.

26. Lebeau J, Lc Chalony C, Prospcri MT, Goubin G. Constitutive overexpression of a $89 \mathrm{kDa}$ heat shock protein in the HBL human mammary cell line converted to a tumorigenic phenotype by the EJ/T24 Harveyras oncogene. Oncogene 1991; $6: 1125-32$.

$m / s n^{\circ} 7$, vol. 8, seplembre 92
27. Hirayoshi $\mathrm{K}$, Kudo $\mathrm{H}$, Hajime $\mathrm{T}$, et al. HSP47 : a tissuc-specific, transformationsensitive, collagen-binding heat shock protein of chicken cmbryo fibroblasts. Mol Cell Biol $1991 ; 11: 4036-44$

28. La Thanguc NB, Latchman DS. Nuclcar accumulation of a heat-shock like protein during hempes simplex virus replication. Biasci Rep $1987 ; 7$ : 475-84.

29. Pallas DC, Morgan W, Roberts TM. The cellular proteins which can associate spccifically with polyomarivus middle $T$ antigen in human 293 cells include the major human 70-kilodalton heat shock proteins. J Virol 1989 ; 63 : 4533-9.

30. Sawai ET, Butcl JS. Association of a cellular heat shock protcin with simian virus 40 large $\mathrm{T}$ antigen in transformed cells. $J$ Virol $1989 ; 63: 3961-73$.

31. May E, Brcugnot C, Duthu A, May P Immunological evidence for the association between simian virus $40115 \mathrm{kd}$ super $\mathrm{T}$ antigen and HSP70 proteins in rat, monkey, and human cells. Virology 1991; 180: 285-93.

32. White E, Spector D, Welch WJ. Diffcrential distribution of the adenovirus E1A protcins and colocalization of E1A with the 70-kilodalton heat shock protein in infected cclls. $J$ Virol 1988 ; 62 : 4153-66.

33. Lim MY, Davis N, Zhang JY, Bose HR The $v$-rel oncogen product is complexed with cellular proteins including its proto-oncogene product and heat shock protcin 70 . Virology $1990 ; 175: 149-60$

34. Pinhasi-Kimhi O, Michalovitz D, BenZecv A, Oren M. Specific interaction between the p53 cellular tumor antigen and major heat shock proteins. Nature 1986 ; 320 : 182-4.

35. Soussi T, Caron de Fromentel C, May P. Structural aspects of the p53 protein in relation to gene cvolution. Oncogene $1990 ; 5$ : 945-52.

36. Koskinen PJ, Sistonen G, Evan RI Morimoto RI, Alitalo K. Nuclear colocalization of cellular and viral myc proteins with HSP70 in myc-expressing cells. J Virol 1991 ; $65: 842-51$

37. Lam KT, Calderwood SK. HSP70 binds specifically to a peptide derived from the highly conserved domain (I) region of p53. Biophys Biochem Res Commun 1992; 184 : 167-74.

38. Davidoff A, Iglchardt JD, Marks JR. Immune response to $\mathrm{p} 53$ is dependent upon p53/HSP70 complexes in breast cancers. Proc Natl Acad Sci USA 1992; 89 : 3439-42.

39. Nagel DC, Picrce SK. A casc for chaperones in antigen processing. Immunol Today $1992 ; 13: 86-9$. 